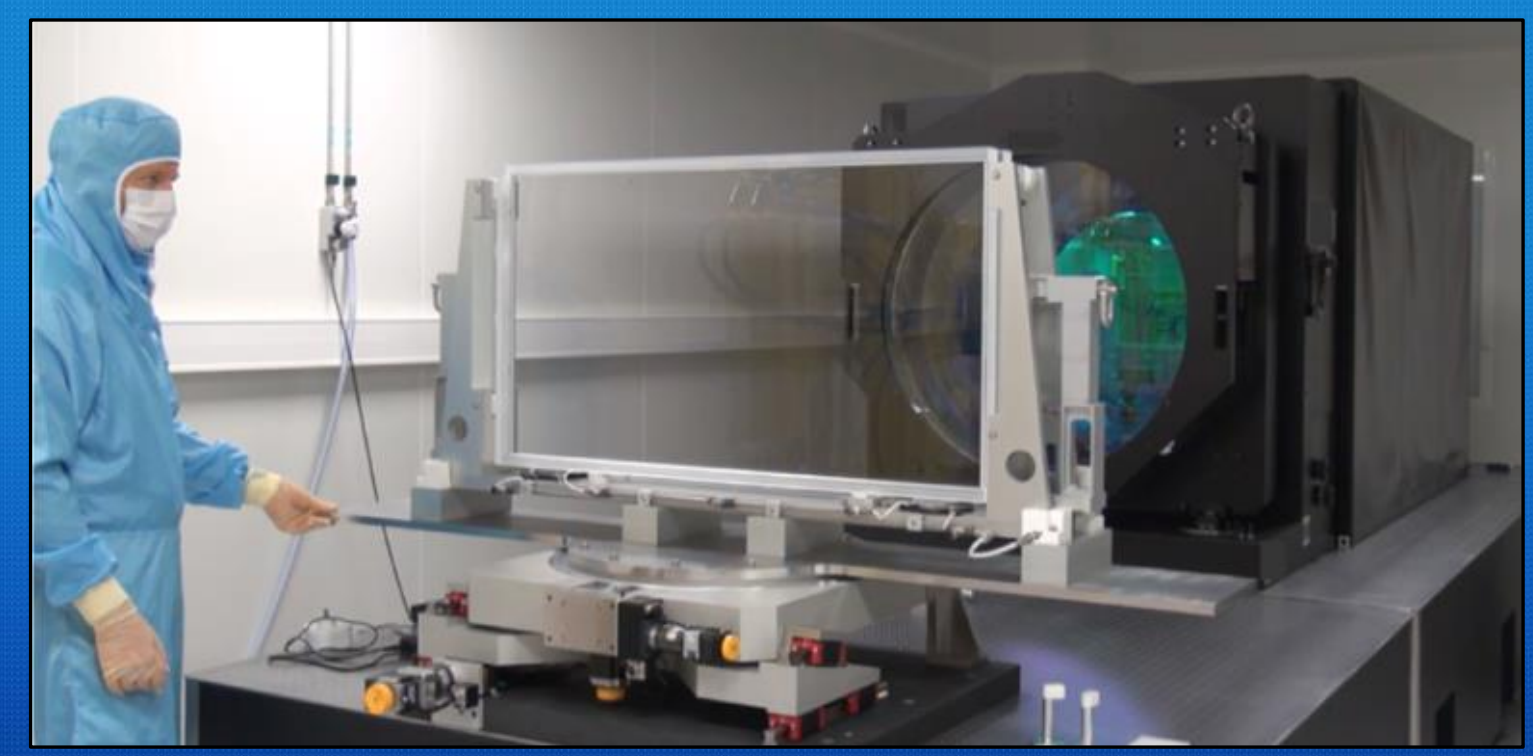

HORIBA

Explore the future
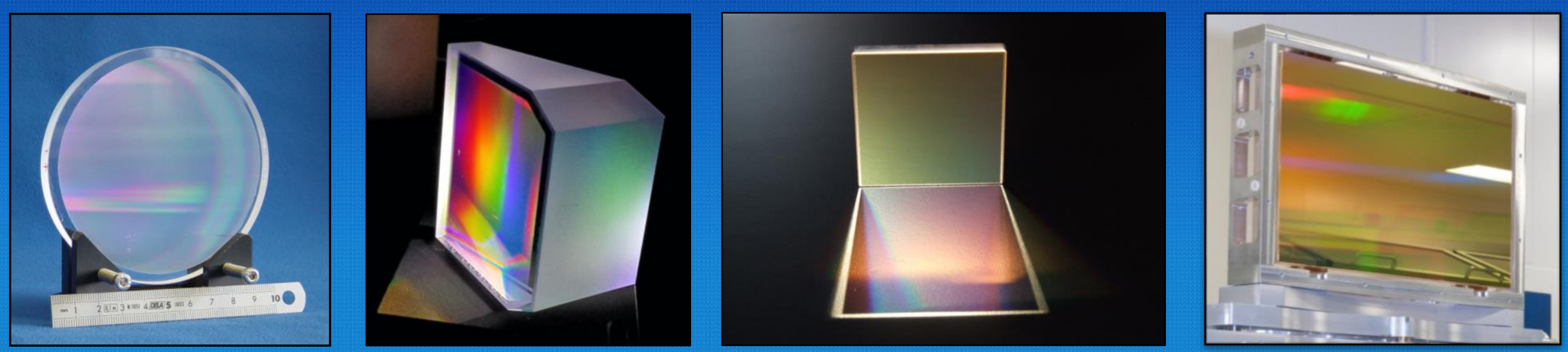


\section{UV Ruled Grating for the Mars Atmosphere and Volatile EvolutioN (MAVEN) mission}

HORIBA

Dr. Arnaud COTEL - HORIBA Scientific arnaud.cotel@horiba.com

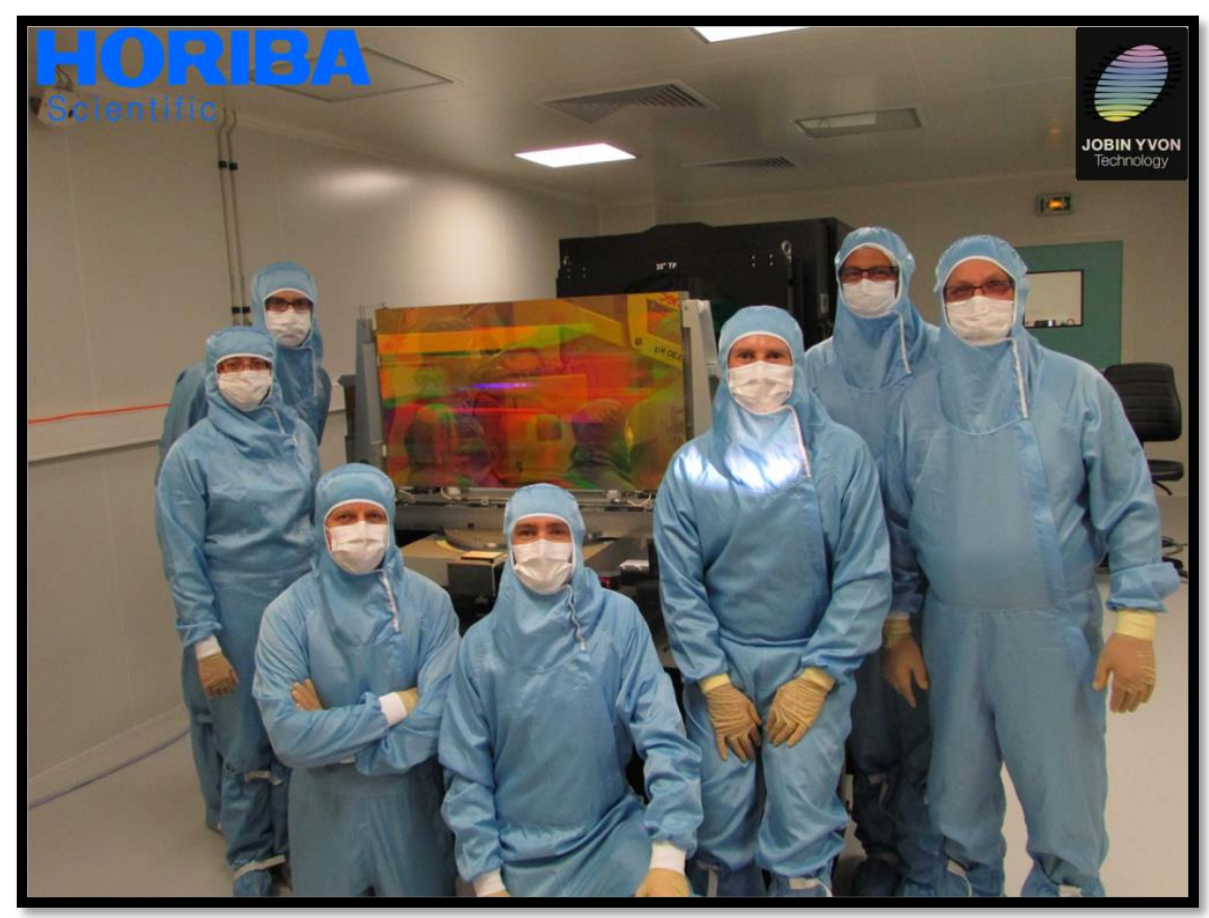

$1968-2018$ : 50 years of Holography at Jobin Yvon $1819-2019$ : 200 years of Jobin Yvon company 


\section{Outline}

- Ruled Gratings capabilities and heritage of Space flight missions

UV Ruled Grating design for the MAVEN Mission

- MAVEN Ruled Grating characterization and performances 


\section{Outline}

\section{- Ruled Gratings capabilities and heritage of Space flight missions}




\section{Longjumeau Grating Facility}

- Historical Jobin Yvon Gratings facility.

- Holographic recording setups up to $600 \mathrm{~mm}$ in clean room to produce Master Gratings.

- Ion-Etchers and Ruling Machines.

- Metrology : efficiency, interferometer, AFM, groove density, ...
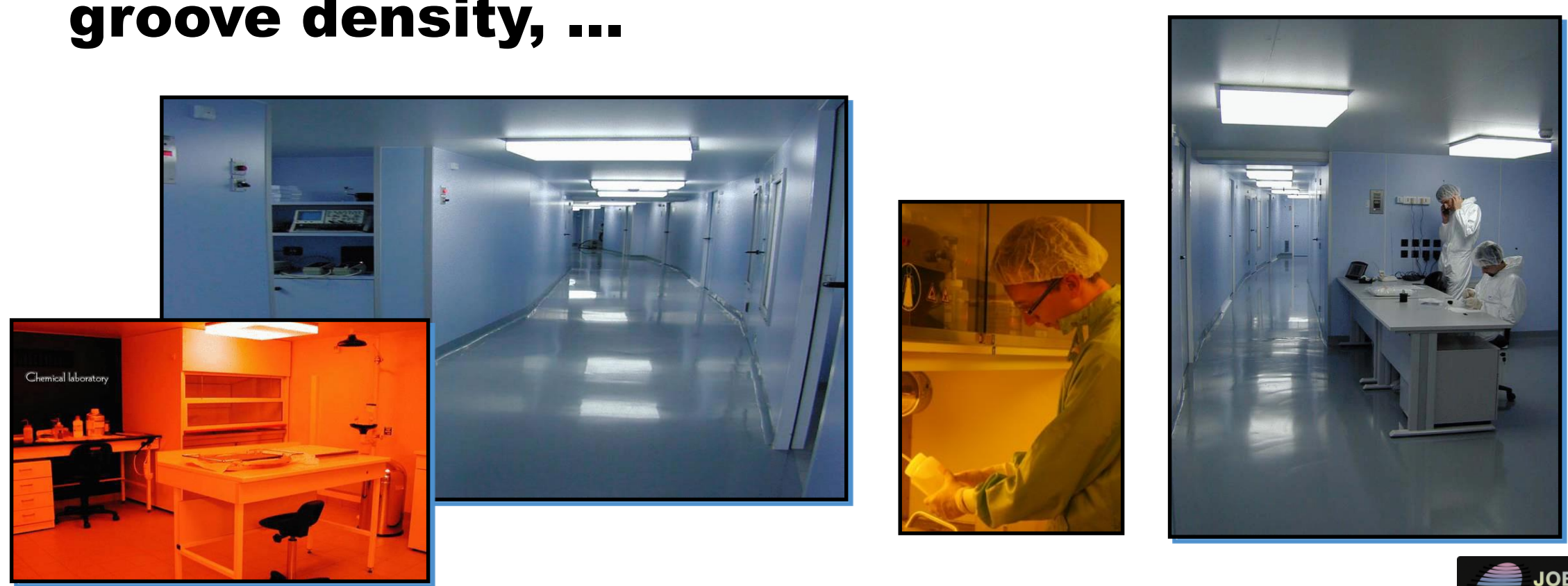


\section{Palaiseau Grating Facility}

- Built in 2012 as the HORIBA Scientific R\&D center in Europe.

- Holography up to $1500 \mathrm{~mm}$ to produce Gratings for CPA High Intense lasers*

- Metrology : AFM, WFE, efficiency.
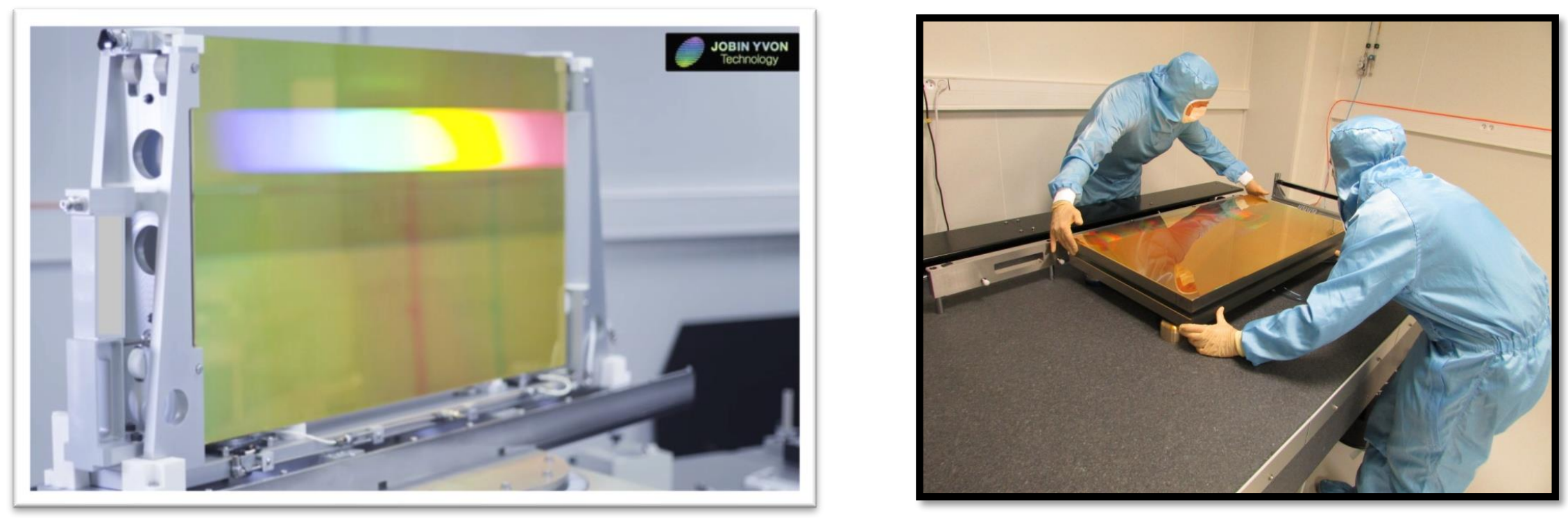

*Donna Strickland and Gérard Mourou, «Compression of amplified chirped optical pulses", Opt. Comm. 56 (1985) => 2018 NOBEL PRIZE in Physics. 


\section{HORIBA Ruled gratings}

Diamond ruling :

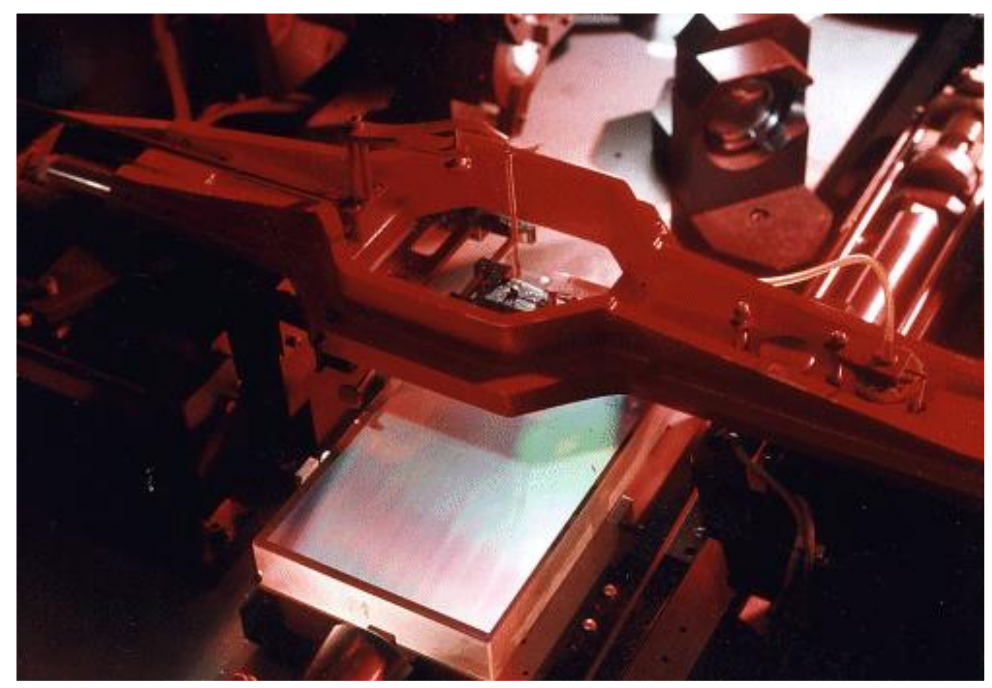

Diamond element directly engraves the groove profile which is blazed.

\section{KEY FEATURES :}

- Low groove density/Echelle

- Ghost-free and low stray light with controlled process

- Small blaze angle possible $\sim 3^{\circ}$ on flat substrate

- Wavelength from UV to IR - Master or Replica gratings possible (TRL9)

- Large choice of substrate materials : Glass, Metal, SiC 
Mid-IR Ruled blazed plane reflection grating

Juno (NASA) mission with the Jovian Infra-red Auroral Mapper (JIRAM) instrument,

- Low blazed angle ruled master gratings ;

- Spectral range : Mid-IR 2-5 $\mu \mathrm{m}$;

- Groove density : $\mathbf{3 0} \mathrm{gr} / \mathrm{mm}$;

- Plane shape ;

- Space flight environmental conditions qualified (thermal cycling from $-173^{\circ} \mathrm{C}$ to $\left.+70^{\circ} \mathrm{C}\right)$. 


\section{Mid-IR Ruled blazed plane reflection grating}

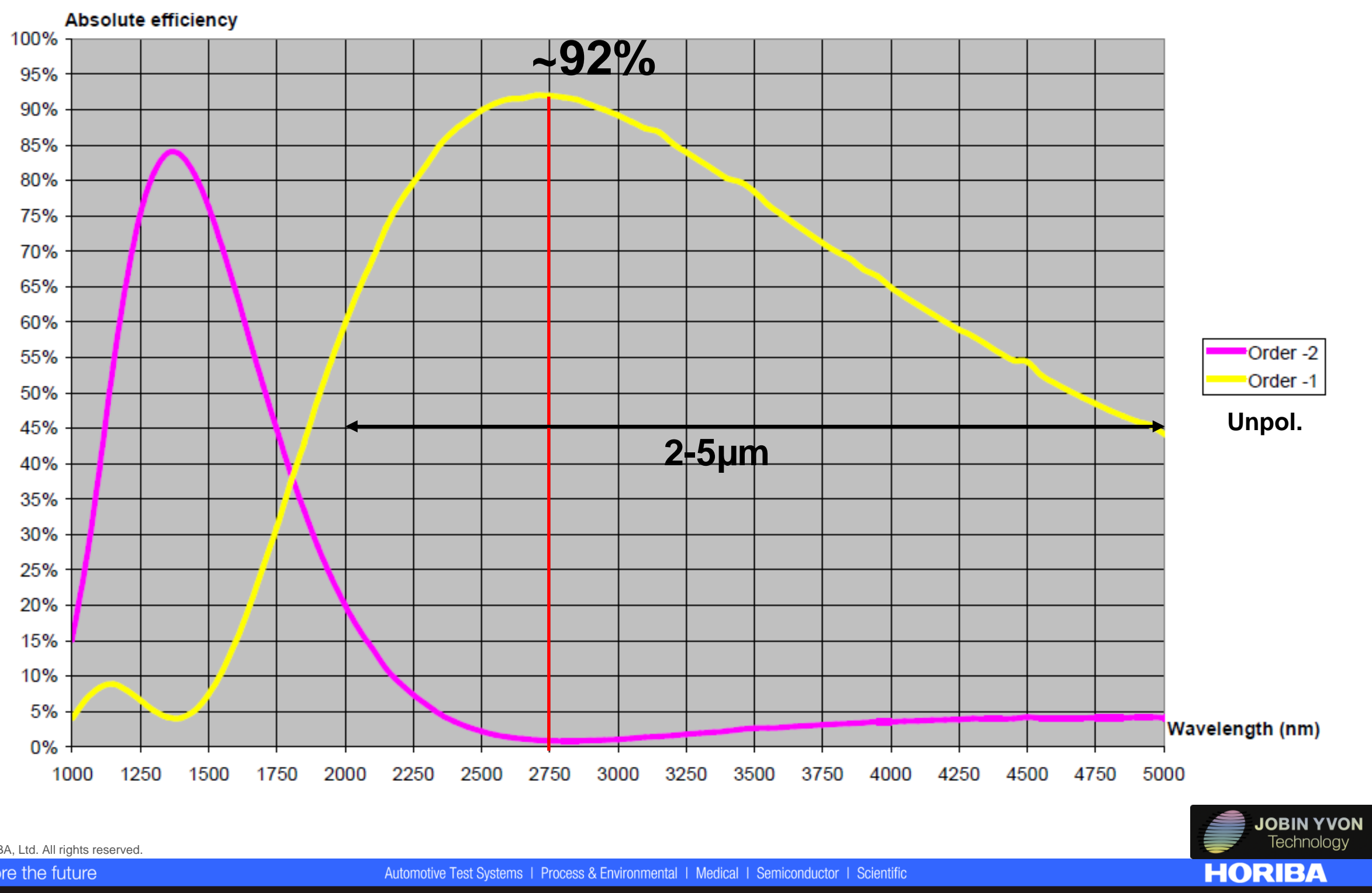




\section{ESA-JAXA Bepi Colombo Mercury нов mission - VIHI instrument}

Broadband 400-2500nm plane ruled master grating with low groove density

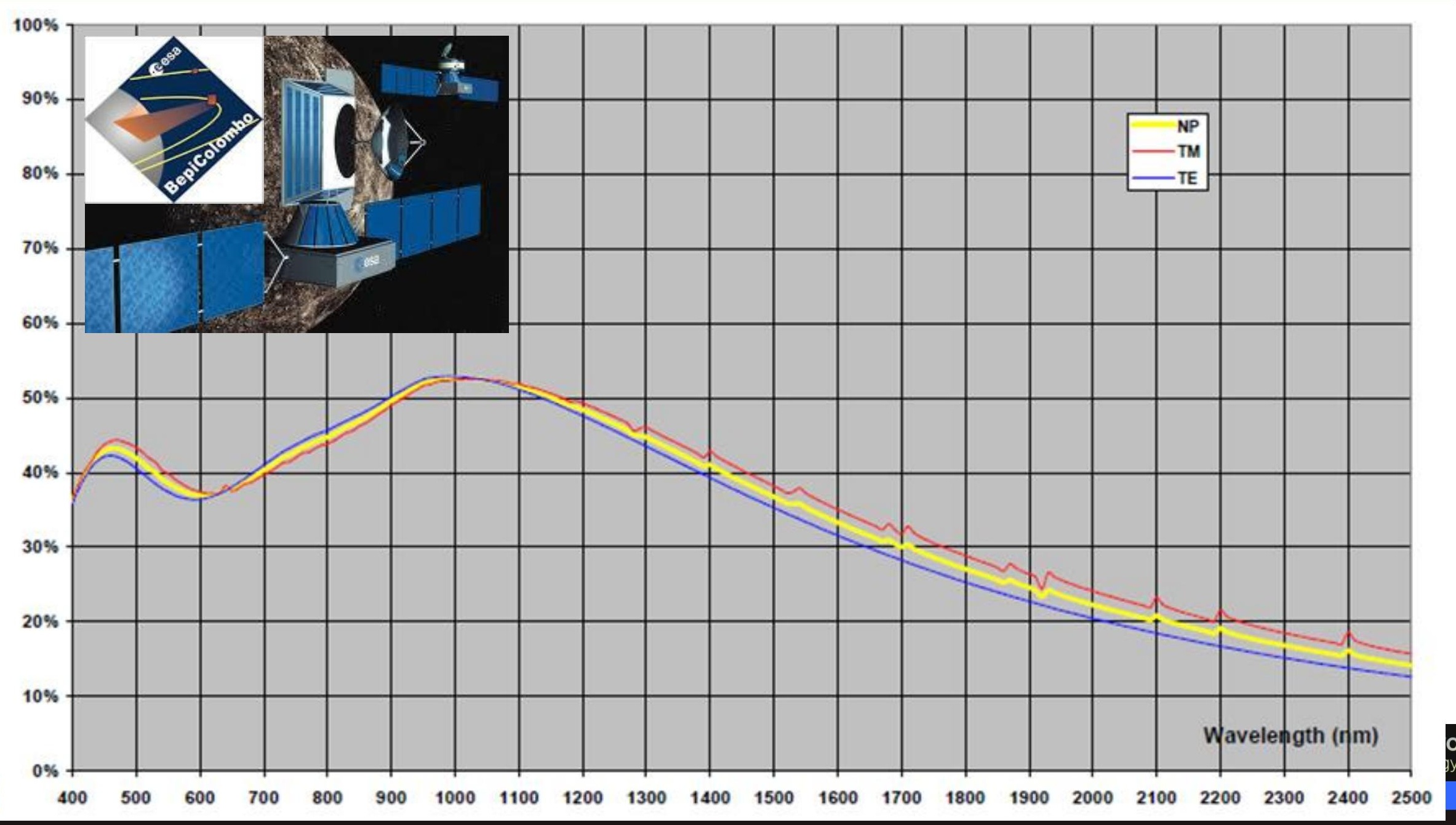




\section{Outline}

\section{- Ruled Cratings capabilities and heritage}

of Space filigt missions

\section{UV Ruled Grating design for the MAVEN Mission}

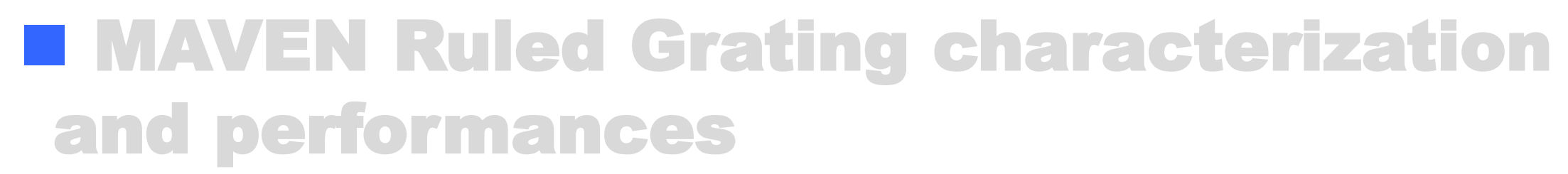




\section{Mars Atmosphere and Volatile EvolutioN (MAVEN) mission}

MAVEN, with its Imaging UV Spectrometer, part of NASA's Mars Scout program, launched in 2013: explores the Red Planet's upper atmosphere, ionosphere and interactions with the sun and solar wind.
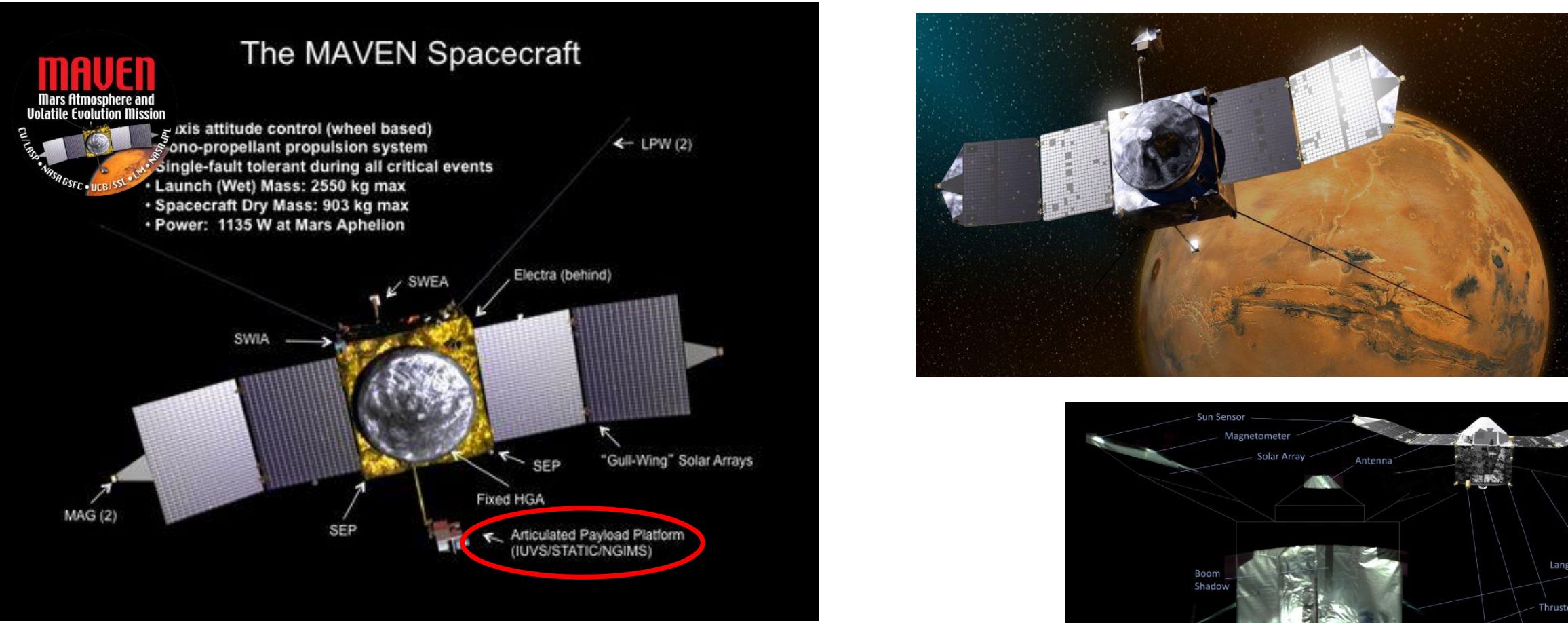

Selfie from IUVS Sept. 2018

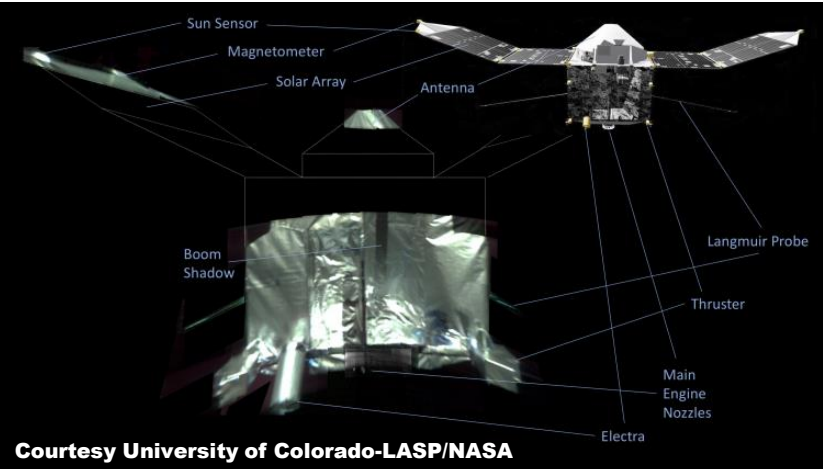


UV Ruled Master Grating design for НОРЕА MAVEN

Blazed ruled plane grating with maximized efficiency in 1st order @280nm AND in the $2^{\text {nd }}$ order @160nm

- Groove density : $276 \mathrm{gr} / \mathrm{mm}$

- Dimensions : H116 x L72mm ;

- Replicated from a master ruled grating ;

- Low grooves roughness to minimize the stray light in the UV;

- Special Al+MgF2 coating.

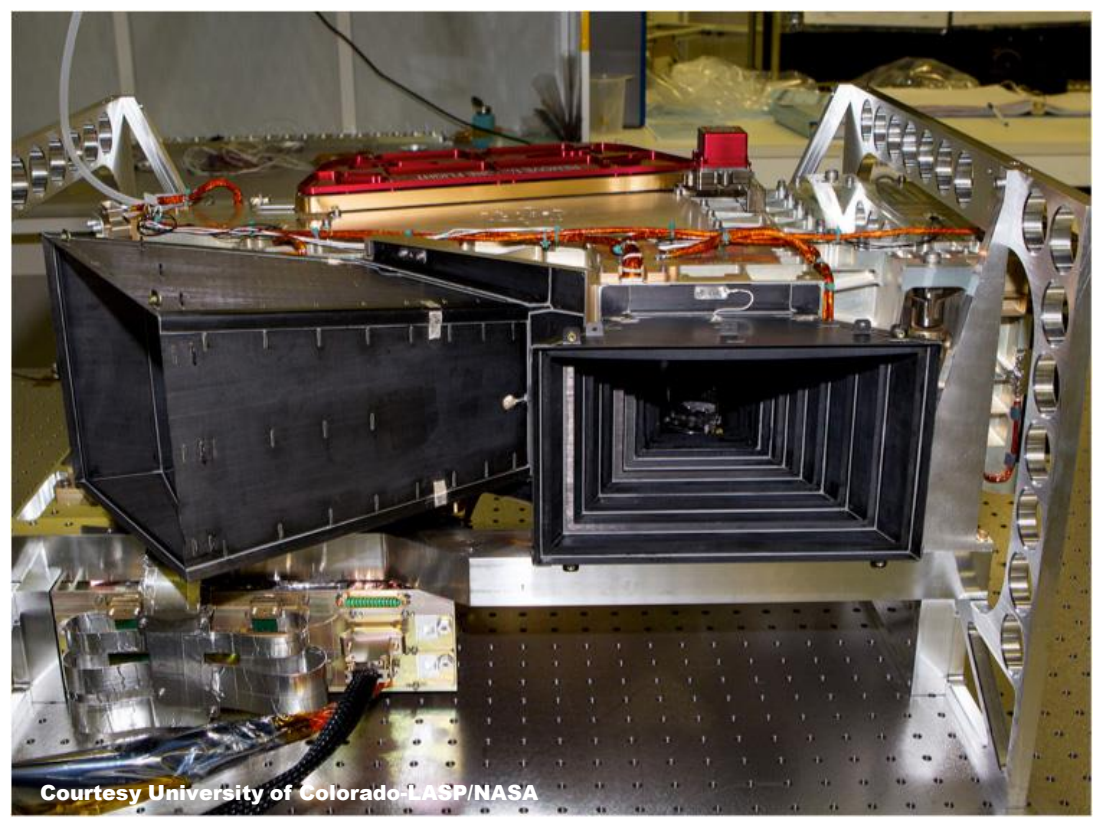

Imaging UV Spectrometer 


\section{MAVEN Grating efficiency \\ simulations / unpolarized, $A O I=3,6^{\circ}$}

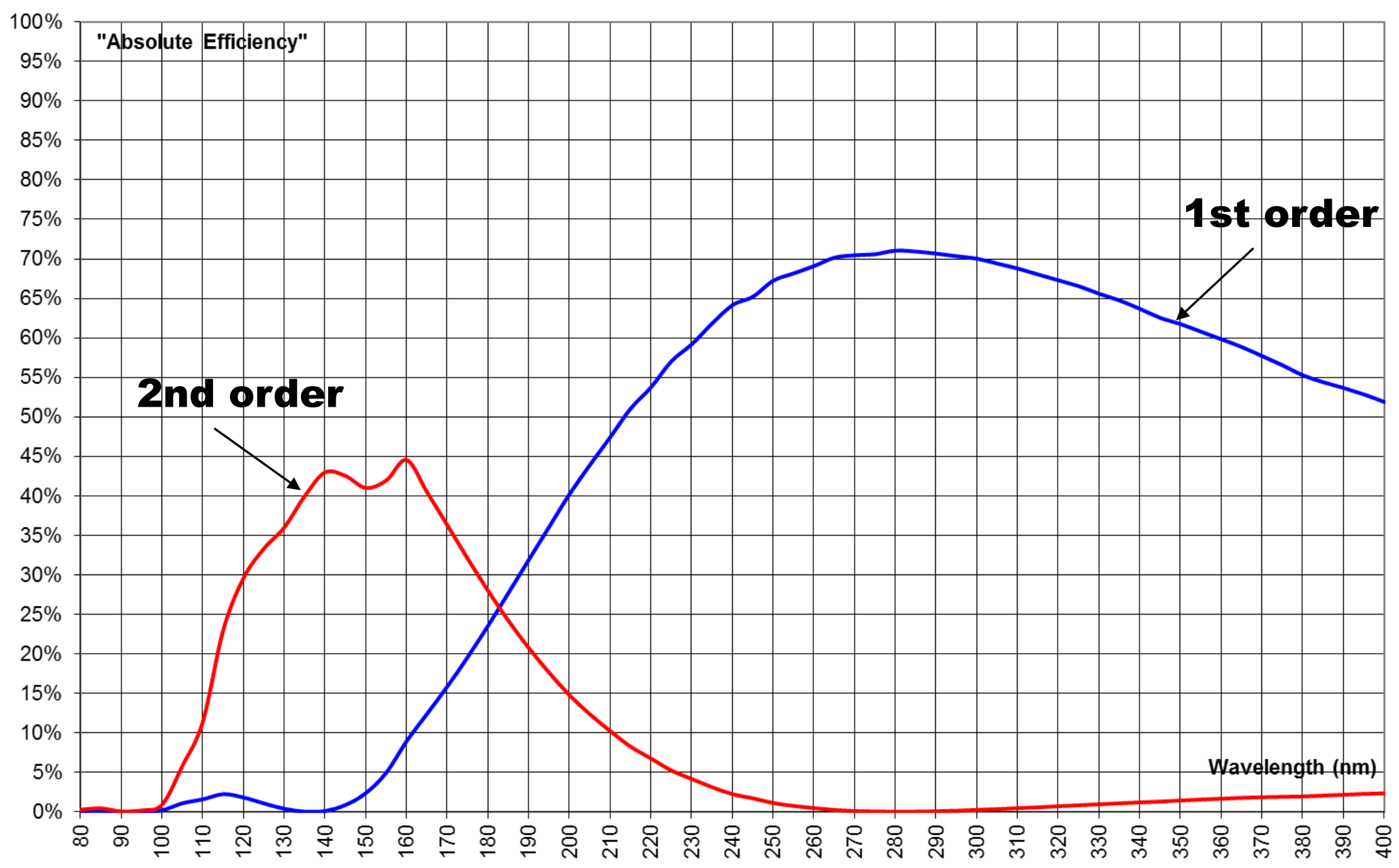




\section{Outline}

and performances 


\section{MAVEN Grating groove profile measurement with AFM}

Grating groove profile measurement (blazed angle, depth) to re-compute the efficiency of the $2^{\text {nd }}$ order $+$

Grooves roughness measurement

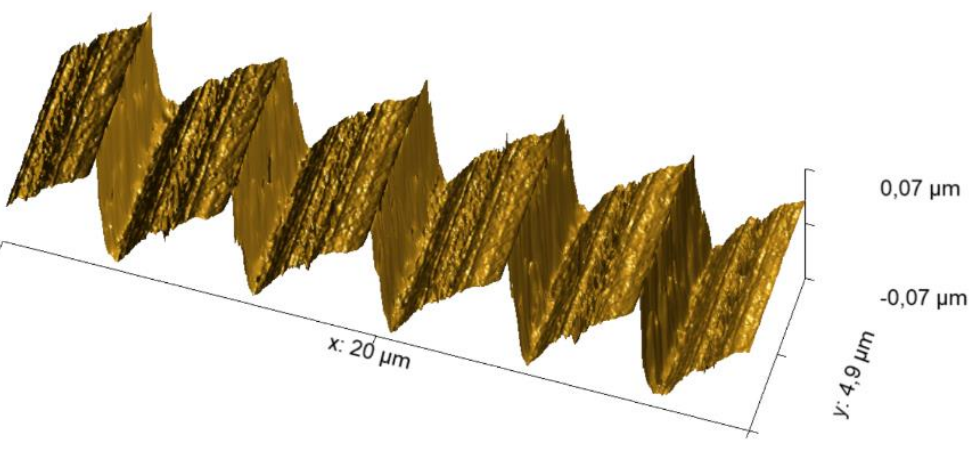

AFM groove profile of MAVEN blazed replica grating
Groove roughness measured at 2,35nm (less than Diamond Turning gratings) 


\section{Stray light characterization of a MAVEN type UV ruled grating}

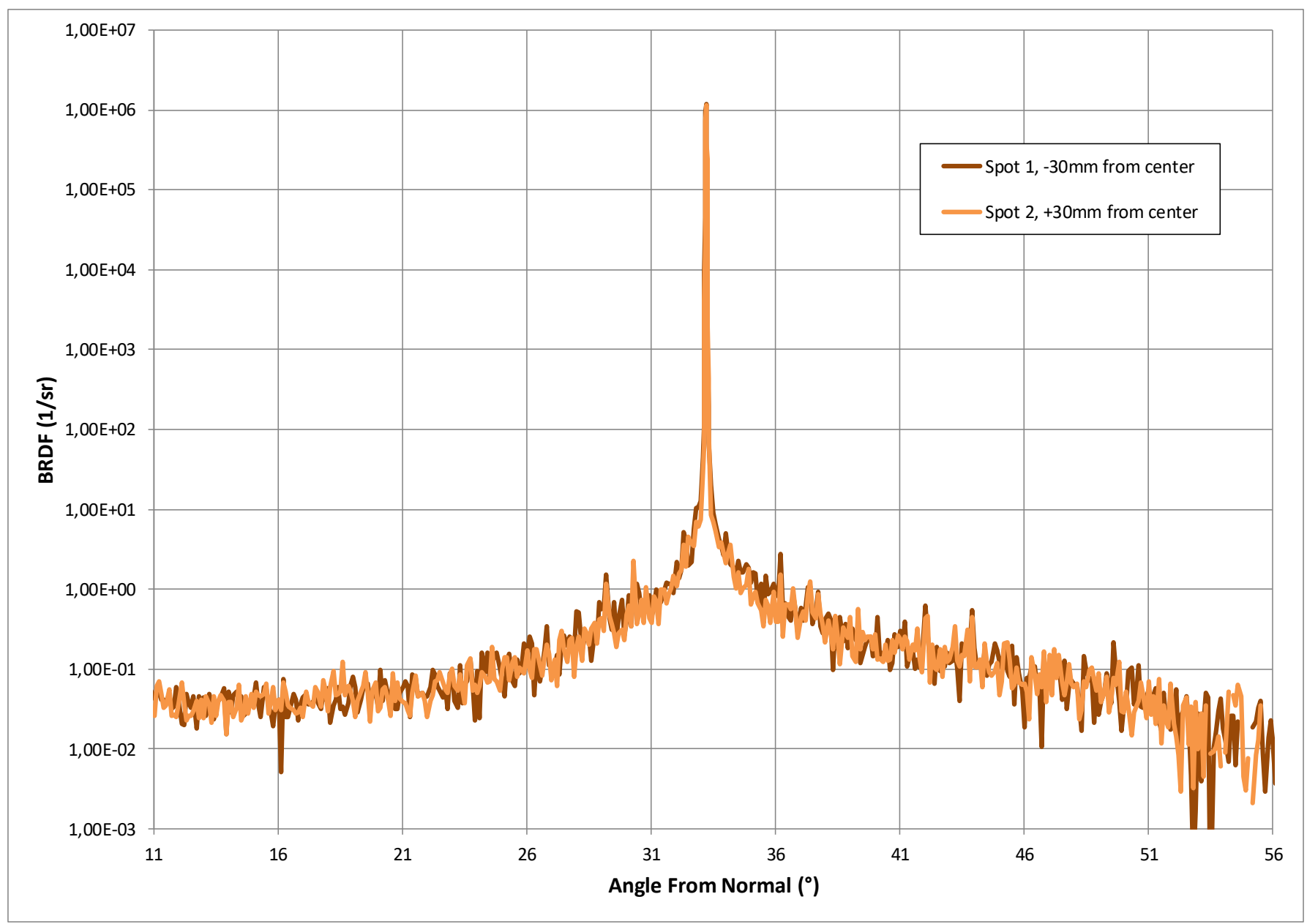

=> No ghosts or parasit orders 


\section{MAVEN Grating Wavefront Error (WFE) measurements}

Surface Data

Fizeau Interferometer measurement @632nm over the whole useful area :

H114xL70mm (grooves parallel to this the height)

$=>\lambda / 5$ PV (w/o power)

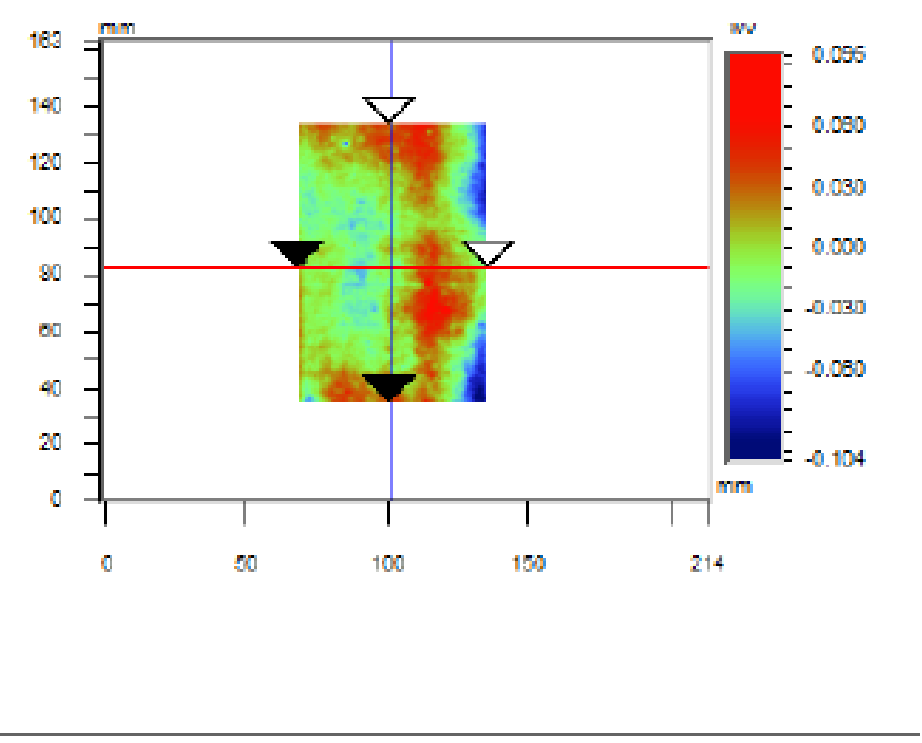

$\begin{array}{ll}\text { PV } & 0.20 \mathrm{wv} \\ 20 \mathrm{pts} \text { PV } & 0.16 \mathrm{wv} \\ \text { RMS } & 0.03 \mathrm{wv}\end{array}$




\section{Conclusion}

We have successfully manufactured the UV Ruled replica Gratings for the IUVS of MAVEN mission.

- Large size $116 \times 72 \mathrm{~mm}$ grating with 276gr/mm groove density.

- $60 \%$ peak efficiency at $280 \mathrm{~nm} / 2,35 \mathrm{~nm}$ grooves roughness, ghost free. 


\section{Thanks for your attention}

\section{HORIBA}

Scientific 